\title{
Vision Based Haptic Multisensor for Manipulation of Soft, Fragile Objects
}

\author{
Artashes Mkhitaryan \\ Machine Vision and Perception Group \\ Technical University of Munich \\ Munich, Germany 80339 \\ Email: mkhitary@in.tum.de
}

\author{
Darius Burschka \\ Machine Vision and Perception Group \\ Technical University of Munich \\ Munich, Germany 80339 \\ Email: burschka@in.tum.de
}

\begin{abstract}
We present a vision based multisensor that is designed for robot interaction with small, soft, and possibly fragile objects. The sensor consists of a rubber membrane, a rectangular frame on which the membrane is mounted and a CCD camera. The entire system is airtight. Based on the observed deformations of the membrane, we determine the contact area, the integral force acting on the membrane, the 3D force distribution over the membrane, and derive properties of the target object by monitoring the evolution of its deformation. We can distinguish between different types of materials, i.e., solid, soft, amorphous, and determine the speed and nature of their deformation. The sensitivity of the sensor can be adjusted by changing the volume of air within the rectangular frame. We achieved a small noise to signal ratio, which allows us to observe small integral forces in the range of $0.5 \mathrm{~N}$ to $2.5 \mathrm{~N}$, with an average error of $0.04 \mathrm{~N}$.
\end{abstract}

\section{INTRODUCTION}

The sense of touch is one of the five fundamental senses that humans use for perceiving the surroundings. A good tactile sensor can augment the robot's ability to perceive, identify and interact with the environment. Significant improvements can be accomplished in grasping and object exploration, given a tactile sensor that can identify the three dimensional force distribution over the contact surface, the target's shape, softness, and it's response during the interaction. We believe that the current state-of-the-art tactile sensors are specialized in one of the above problems and struggle when presented with the other challenges. For example Damian et. al [1] present an artificial silicon based skin for prosthetic interfaces that allows the estimation of the two dimensional motion over the surface of the skin, but it is not capable of registering complex shapes and deformations. Katsunari [2] et. al. introduced a GelForce surface traction sensor for a robotic hand. While the sensor computes the three dimensional force distribution, it suffers from low resolution [3] and does not provide data regarding the target's shape.

\section{THE SENSOR}

In this Section, we give the full description of our sensor. First, in Sec. II-A we describe it's physical setup. Following in Sec. II-B, we present the algorithm for 3D reconstruction of the surface of the contact area. Last, we outline the computation of forces and their distribution over the contact surface in Sec. II-C.

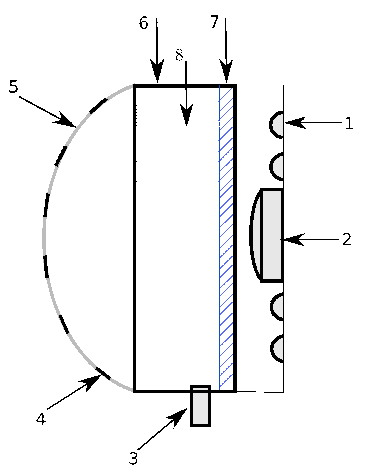

Fig. 1. (1): LED, (2): CCD camera, (3): a valve to regulate the amount of air within the frame, (4): rigid circle markers, (5): rubber skin surface, (6): the airtight frame of the finger,(7): glass, (8): air.

\section{A. Physical Setup}

The design of our multysensor is rather simple. It consists of a thin white rubber membrane that has rigid black circles attached to its surface. The membrane is mounted on a hollow rectangular frame. The other side of the frame is sealed with a glass. The rectangular frame is also equipped with a small valve that allows to control the amount of air within the frame (Fig. 1). By increasing and decreasing the amount of air within the frame we can regulate the shape of the rubber membrane as well as its sensitivity to deformations caused by external forces. From the other side of the glass (outside of the rectangular frame) a CCD camera is mounted combined with a few LEDs to provide lighting. This is done in such way that it is always possible to keep the membrane in the image plane of the camera.

\section{B. 3D Reconstruction of the Surface of the Membrane}

1) Extracting the Circle Centres: It is a well known fact that the projection of a circle is always an ellipse unless the projection surface is parallel to the surface of the circle. Thus, given the image $I_{j}$ captured from camera, we extract all the black ellipses $\left\{e_{i}\right\} \in I_{j}$ (Fig. I) according to [4] that correspond to the projections of the circles $\left\{c_{i}\right\}$. Since an ellipse is a conic section, in the most general case it is possible to write the ellipse equation in this form:

$$
m_{1} x_{i}^{2}+m_{2} x_{i} y_{i}+m_{3} y_{i}^{2}+m_{4} x_{i}+m_{5} y_{i}+m_{6}=0
$$




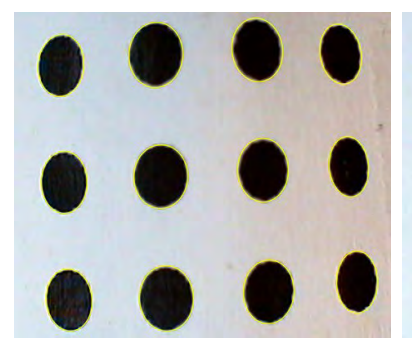

(a) Detected ellipses

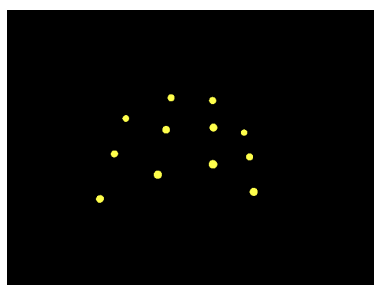

(d) Initial 3D reconstruction

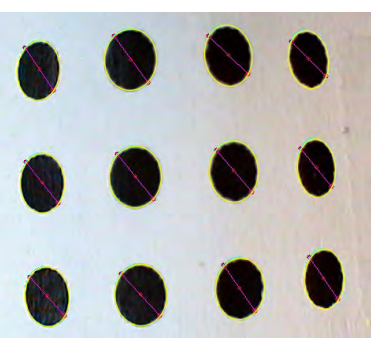

(b) Computed centers of circles

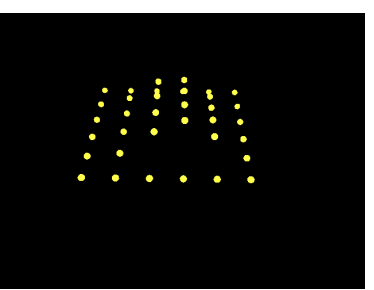

(e) Mashing and bilinear interpolation

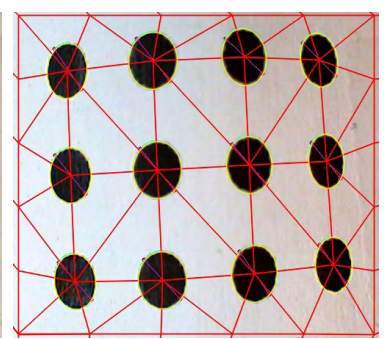

(c) Trinagulation, and mashing

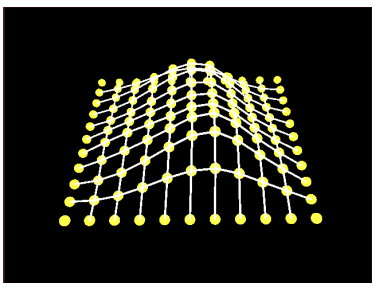

(f) B-spline fitting

Fig. 2. Step by step illustration of the membrane surface reconstruction process.

Where $x_{i}$ and $y_{i}$ are the coordinates of all the points on the considered ellipse. The matrix representation of conic section can be written as following:

$$
M=\left(\begin{array}{ccc}
m_{1} & m_{2} / 2 & m_{4} / 2 \\
m_{2} / 2 & m_{3} & m_{5} / 2 \\
m_{4} / 2 & m_{5} / 2 & m_{6}
\end{array}\right)
$$

Where the matrix elements are the corresponding coefficients from Eq. (1). Since $M$ is a symmetric matrix its eigenvector matrix $V$ will be orthogonal and the following will hold:

$$
M=V * \Lambda * V^{T} \quad \Lambda=\left(\begin{array}{ccc}
\lambda_{1} & 0 & 0 \\
0 & \lambda_{2} & 0 \\
0 & 0 & \lambda_{3}
\end{array}\right)
$$

Here $\lambda_{1}>\lambda_{2}>\lambda_{3}$ are the eigenvalues of $M$. As it is shown in [5] it is possible to estimate the position $P_{i}=\{u, v, 1\}^{t}$ of the projection of the center of the circle $c_{i}$ in the image plane (Fig. 2(b)) by:

$$
P_{i}= \pm V\left(\begin{array}{c}
-\sqrt{-\lambda_{3} / \lambda_{1}} \sin \psi \\
0 \\
\sqrt{-\lambda_{1} / \lambda_{3}} \cos (\psi)
\end{array}\right)
$$

where:

$$
\left.\psi=\arccos \sqrt{(} \frac{\lambda_{2}-\lambda_{3}}{\lambda_{1}-\lambda_{3}}\right)
$$

2) Reconstructuion of the $3 D$ Pose of the Circle: Consider Fig.(II-B.2), here $D$ is the center of the circle, $A$ and $B$ are two points on the circle such that line $A B$ passes through the center of the circle, thus $A B=B A=r$. Where $r$ is the radius of the circle. $\Pi$ is the image plain and $C$ is the camera center, $a, b$, and $d$ are the projections of $A, B$ and $D$ on the image plane correspondingly, hence $a$ and $b$ are located on the ellipse, and $d$ is inside (Fig. 2(b)). We are interested in

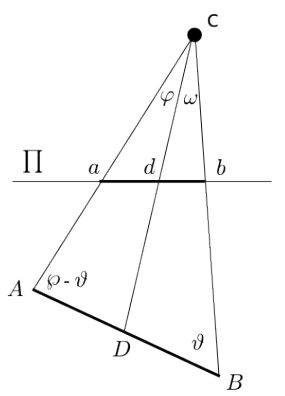

Fig. 3. Here $C$ is the camera center, $\Pi$ is the image plane, $a$ and $b$ are two points on the ellipse, and line $a b$ passes through $d$ that is the projection of circle center $D, A D=D B$, and $\rho=180-\phi-\omega$

computing the length of $C D$ to determine the $3 D$ pose of the circle. This can be computed by applying sine rule:

$$
\begin{gathered}
\frac{\sin (\phi)}{r}=\frac{\sin (\rho-\nu)}{C D} \\
\frac{\sin (\omega)}{r}=\frac{\sin (\nu)}{C D}
\end{gathered}
$$

here $\rho=180-\phi-\omega$. By dividing Eq. (6) by Eq. (7) we obtain:

$$
\frac{\sin (\phi)}{\sin (\omega)}=\frac{\sin (\rho-\nu)}{\sin \nu}
$$

By simplifying Eq. (8) we can derive $\nu$ to:

$$
\cot \nu=\frac{\sin (\phi)}{\sin (\omega) \sin (\rho)}+\cot (\rho)
$$

further we combine (9) and (7) to derive equation for $C D$ :

$$
C D=\frac{r * \sin (\nu)}{\sin (\omega)}
$$


Here the remaining unknowns are the angles $\omega$ and $\phi$, that can be computed from $(\hat{\mathbf{C a}} \cdot \hat{\mathbf{C d}})$ and $(\hat{\mathbf{C d}} \cdot \hat{\mathbf{C b}})$ dot products correspondingly, where ${ }^{\wedge}$ indicates a unit vector. $a$ can be chosen as an arbitrary point on an ellipse, where $b$ will be defined as a point that is located on the intersection of the ellipse and a line that passes through $a$ and $d$. For best accuracy, we suggest to pick the line $a b$ such that it is parallel to the major axis of the ellipse. After the depth $C D$ of the center of the circle is known it is possible to re-project the point into $3 D$ using the inverse of the intrinsic camera matrix, and obtain the unstructured point cloud $\left\{p_{i}\right\}$ (Fig. 2(d)).

3) Mashing and Surface Fitting: Only the point cloud $\left\{p_{i}\right\}$ is not sufficient to extract information regarding the contact area of the sensor. Thus as a next step we preform B-spline surface fitting. The point cloud $\left\{p_{i}\right\}$ is not structured and hence it requires some processing for the surface fitting. We consider the set $\left\{p_{i}\right\}$ of projected circle centres in the image plain and the rectangular profile of the frame, which will be later used as boundary conditions. We assume that the pose of the rectangular profile in reference to the camera center is known. Former can be obtained by running the 3D reconstruction described in the previous chapter with an open valve. First we obtain triangulation $\left\{t_{i}\right\} \in T$ by performing Delaunay triangulation for $\left\{p_{i}\right\}$ (Fig. 2(c)). Next, we generate a $2 D$ low resolution Cartesian grid within the frame, and for each node $v_{i} \in t_{i}$ of that grid, we compute its $3 D$ pose by performing barycentric interpolation in $t_{i}$, using the frame and the points located on the frame as boundary conditions (Fig.2(e)). In the final step, we fit a B-spline surface to the $3 D$ structured point cloud obtained from the grid (Fig.2(f)).

\section{Computation of Force Distribution}

Due to its molecular structure, the rubber is not a Hookean material, i.e. the relation between the starch and the applied force is not linear [6], hence it is not possible to analytically compute the force distribution. To compute the relative force distribution over the surface of the membrane we make some assumptions. First, we assume that the membrane has uniform thickness $(d)$. The thickness is considerably smaller then the width $(w)$ and the length $(l)$ of the membrane $(w \gg d, l \gg d)$. The thinning of the thickness due to starches is neglectful, and the external forces are small, i.e. in the range of $[0.5-$ $2] N$. Based on this assumptions, we claim that the elasticity of the rubber membrane is constant within a small stretch range (proof by experiment Sec. (III)). If the volume of air remains constant within the frame of the sensor the following is true:

$$
\Delta F=c \Delta A
$$

Where $c$ is a constant, $\Delta F$ is the overall change in the external forces acting on the membrane and $\Delta A$ is the change of the area of the membrane due to $\Delta F$. Form (11) and the above made assumptions it follows that the membrane is stretching uniformly. Therefore, to compute the direction and magnitude of forces over the entire surface we can approximate the membrane by a grid of springs (Fig. 2(f)).

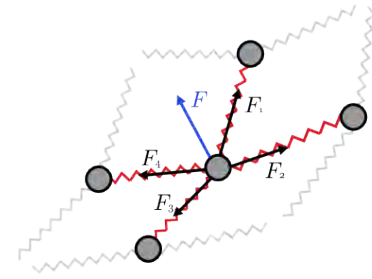

Fig. 4. Computation fo the force acting on a node. Here $\mathbf{F}=\mathbf{F}_{\mathbf{1}}+\mathbf{F}_{\mathbf{2}}+$ $\mathbf{F}_{3}+\mathbf{F}_{4}$

Since the surface is stretching uniformly, each node of the grid will be equidistant to its neighbours. Thus the direction and magnitude of force at that node will only depend on the curvature of the surface at that point (Fig. 4).

\section{RESULTS}

The 3D reconstruction of the contact area is computed based on the 3D positions of the circle markers on the membrane (Fig.1). Examples of the 3D reconstructions with corresponding force distributions are illustrated in Fig.7. We generated 15 synthetic images (Fig. 6(a)) each containing 16 ellipses that represent the projection of the circle markers with known poses, to evaluate the accuracy of the vision system. We achieved an average error for the circle marker pose estimation of $0.39 \mathrm{~mm}$ (Fig. 6(b),6(c)). Since the rubber is not a Hookean material the force to strain relation is generally nonlinear, thus it is not possible to analytically compute force from strain. However we claim that for small strains the Hook's model holds. To support this, we conducted an experiment where we attached a force sensor to our sensor and drove a cylindrical solid object into the rubber membrane with $1 \mathrm{~mm}$ increments. For each increment, we registered the force from the force sensor and the estimated integral force acting on the membrane. Fig.5(a) depicts that there is a linear correspondence between the measured and the relative forces. The overall average error for these measurements is $0.04 \mathrm{~N}$. Relative integral force to deformation ratio for a similar experiment conducted on a solid cylinder and a soft sponge is illustrated in Fig.5(b). Note that for sponge the force to deformation curve is parabolic, and by fitting a curve to the datapoints it is possible to determine the stress/strain relation. Fig.5(c) illustrates the relative integral force profile over time for the case when the sensor is continually driven into the same objects.

\section{CONCLUSION}

We have presented a vision based haptic multisensor that is aimed to interact with soft and fragile objects. Our sensor is capable of determining the shape of the target object as well as the forces and their distribution over the contact area. It can detect the dynamic changes of the target object and determine the nature of its deformation. 


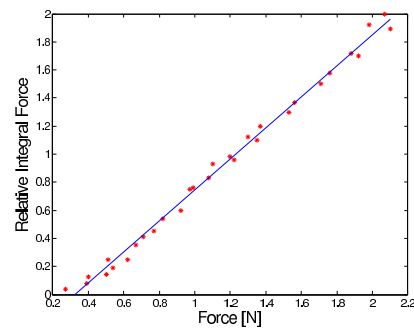

(a) Relative Integral Force/Measured Force Profile.

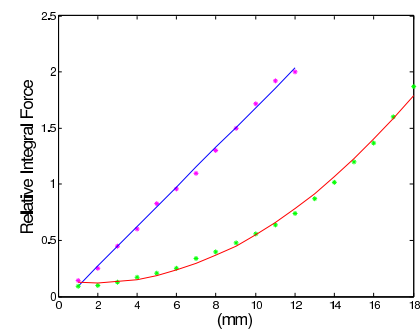

(b) Force/Deformation Profile.

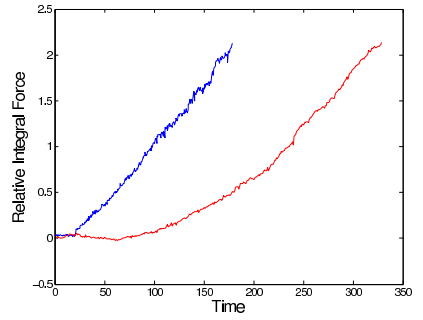

(c) Force/Time Profile.

Fig. 5. Here the blue and red lines correspond to the sold cylinder and soft sponge respectively.

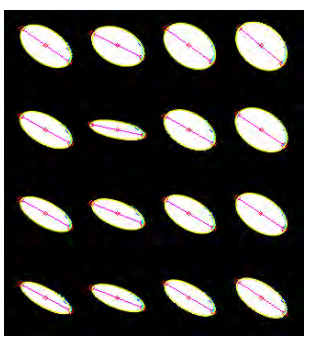

(a)

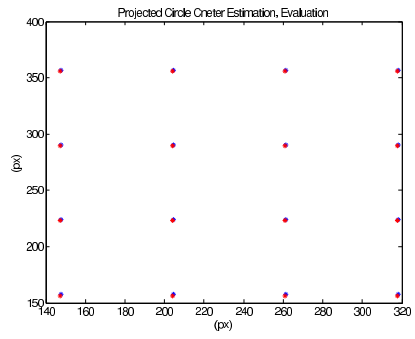

(b)

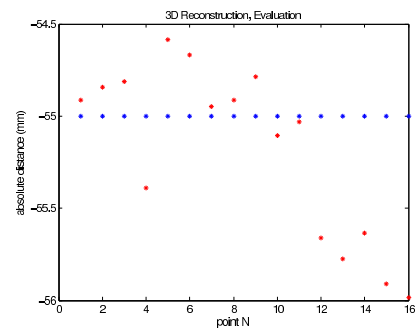

(c)

Fig. 6. 6(a) Illustrates the generated image that contains the projections of circles with known poses. In 6(b) are plotted the estimated (red) and known (blue) circle center projections, and correspondingly the absolute distances from the camera center are illustrated in 6(c).

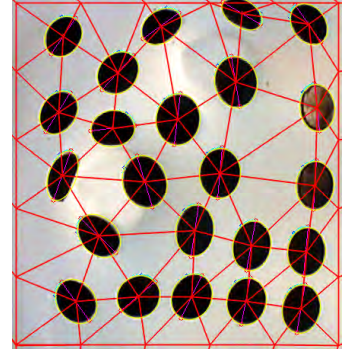

(a)

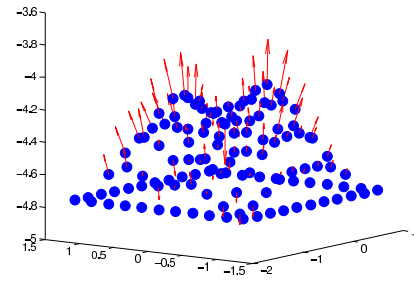

(d)

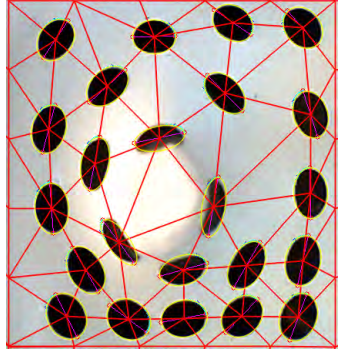

(b)

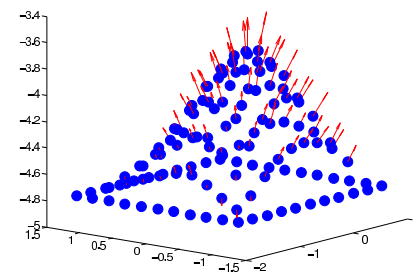

(e)

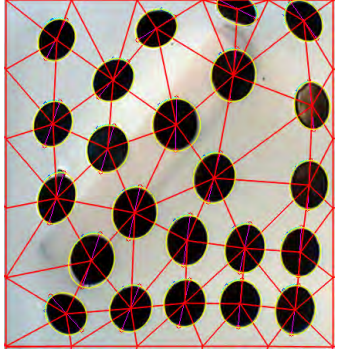

(c)

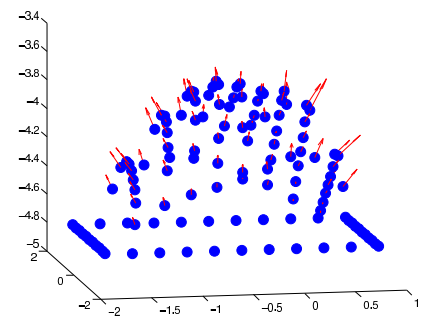

(f)

Fig. 7. 7(a),7(b),7(c) Illustrate the captured and processed images from the CCD camera for three different objects, and 7(d),7(f),7(f) the respective 3D reconstructions, and force distributions. Here the force vectors are illustrated with a minus sign, for visualization purposes.

\section{ACKNOWLEDGEMENT}

This work has been supported by an internal grant "RealTime Perception and Exploration with Collaborating Agents" of the German Aerospace Center (DLR).

\section{REFERENCES}

[1] Damian et. al. "Artificial Ridged Skin for Slippage Speed Detection in Prosthetic Hand Applications", IEEE/RSJ IROS, 2010

[2] Katsunari et. al. "Finger-Shaped GelForce: Sensor for Measuring Surface Traction Fields for Robotic Hand" IEEE Trnas. on Haptics, 2010
[3] Chorley et. al. "Development of a Tactile Sensor Based on Biologically Inspired Edge Encoding", ICAR, 2009.

[4] Suzuki, S. and Abe, K., Topological Structural Analysis of Digitized Binary Images by Border Following. CVGIP 30 1, pp 32-46 (1985)

[5] Philip, Johan "An algorithm for determining the position of a circle in 3D from its perspective 2D projection ", Technical report. KTH, Stockholm.

[6] D.R. Merritt and F. Weinhaus "The pressure curve for a rubber balloon" American Journal of Physics, 1978. 